\title{
Jackiw-Teitelboim gravity and the models of a Hawking-Page transition for 2D black holes
}

\author{
Arindam Lala* \\ Instituto de Física, Pontificia Universidad Católica de Valparaíso, Casilla 4059, Valparaiso, Chile \\ Hemant Rathi ${ }^{\dagger}$ and Dibakar Roychowdhury \\ Department of Physics, Indian Institute of Technology Roorkee, Roorkee 247667, Uttarakhand, India
}

(Received 14 August 2020; accepted 14 October 2020; published 9 November 2020)

\begin{abstract}
We propose a top down construction for Jackiw-Teitelboim (JT) gravity using compactification of $D=5$ gravity theories in the presence of Abelian $[U(1)]$ as well as $S U(2)$ Yang-Mills fields. The background solutions corresponding to the $D=2$ model have been obtained in the perturbative regime of the theory where the corrections have been estimated over the uncharged JT solutions while treating the gauge couplings as the parameters of the expansion. Our analysis reveals the existence of two classes of solutions, namely, (i) the interpolating vacuum solution with $\mathrm{AdS}_{2}$ in the IR and Lifshitz 2 in the UV (which serves as the thermal radiation background for our analysis) and (ii) the charged 2D black hole solution exhibiting Lifshitz ${ }_{2}$ asymptotics. The analysis on thermal stability reveals the onset of a first-order phase transition at $T \sim T_{0}$ such that for $T<T_{0}$ the only possible state is the thermal radiation background without any black hole. On the other hand, as the temperature is gradually increased beyond a certain crossover value $T \sim T_{2}\left(>T_{0}\right)$, an emerging globally stable black-hole-dominated phase has been observed which clearly indicates the onset of a Hawking-Page transition in 2D gravity models.
\end{abstract}

DOI: 10.1103/PhysRevD.102.104024

\section{OVERVIEW AND MOTIVATION}

In today's literature, the celebrated AdS/CFT correspondence $[1,2]$ is widely accepted to be the most remarkable achievement that took place in modern theoretical physics during past two decades. The underlying mechanism that stands behind this proposal is based on the so-called holographic principle [3], whose primary focus is to understand the strong coupling dynamics in large $\mathrm{N}$ quantum field theories using a dual (weakly coupled) stringy or gravitational description living in higher dimensions. Since its discovery, several interesting examples have been discovered as well as tested within the realm of strong/ weak conjecture, among which the very recent discovery of an interesting toy model of quantum holography [4,5] is something worthy of praise. This proposal is based on the

\footnotetext{
*arindam.physics1@gmail.com arindam.lala@pucv.cl

thrathi07@gmail.com hrathi@ph.iitr.ac.in

*dibakarphys@gmail.com dibakar.roychowdhury@ph.iitr.ac.in

Published by the American Physical Society under the terms of the Creative Commons Attribution 4.0 International license. Further distribution of this work must maintain attribution to the author(s) and the published article's title, journal citation, and DOI. Funded by SCOAP.
}

original work of Ref. [6] and, therefore, goes under the name of Sachdev-Ye-Kitaev (SYK) models [7,8].

Ever since this proposal has been put forward, there has been growing interest in order to explore the large $\mathrm{N}$ nearinfrared (IR) dynamics in SYK models using its dual gravitational description(s) that lives in one higher dimension. The collective analyses unveil $(1+1)$ D JackiwTeitelboim (JT) dilaton gravity as the dual model $[9,10]$ that has been conjectured. Putting all these pieces together finally leads toward an emergent SYK/AdS $\mathrm{A}_{2}$ correspondence [11-27]. These analyses have been subsequently extended for charged SYK models and their corresponding dual gravitational counterparts [28-30].

The purpose of the present work is to elaborate an example of dimensional compactification which finally leads toward $(1+1) \mathrm{D}$ JT dilaton gravity in the presence of nontrivial matter (gauge) couplings. The parent 5D model $[31,32]$, that we choose to start with, was actually proposed in order to construct electrically charged spacetime solutions with anisotropic Lifshitz scaling [33,34]. The Chern-Simons (CS) term in the original 5D model [31] was actually a supergravity-inspired " $F F A$ "-type term $[31,35]$. However, the dimensional reduction, on the other hand, results in some form of the $D=2 \mathrm{JT}$ model where both gravity as well as the dilation are found to be nonminimally coupled with the Abelian $[U(1)]$ and the $S U$ (2) Yang-Mills sectors of the theory. 
Below, we enumerate some notable facts about the $D=2$ model that is constructed in this paper.

(i) The ground state (vacuum) of the theory has been found to be interpolating between a Lifshitz 2 at UV and an $\mathrm{AdS}_{2}$ in the IR. This we identify as the thermal radiation background for our subsequent analysis in the Euclidean formalism.

(ii) Thermal excitations have been identified as charged black holes with Lifshitz 2 asymptotics. In the Euclidean framework, these black holes serve as the basis for what we call the analog of HawkingPage (HP) transition [36-41] in 2D gravity models.

(iii) Considering the Euclidean framework, we perform our analysis of thermal stability in black holes using a canonical ensemble. Our analysis reveals the existence of a certain critical temperature $T \sim T_{0} \sim$ $\sqrt{\mu_{0}}$ such that for $T>T_{0}$ there exist two possible phases of black holes - one with lower mass and a negative heat capacity and the other with higher mass and a positive heat capacity. As the temperature of the system is reduced below $T_{0}$, the black hole phase with negative heat capacity decays into pure thermal radiation via a first-order phase transition at $T=T_{0}$. On the other hand, as the temperature is increased above $T_{0}$, the lower mass black hole gradually transits into a globally stable phase of larger mass black hole that is in thermal equilibrium with its surrounding radiation background. During this course of transition, we observe several transition temperatures $\left(T_{0}<T_{1}<T_{2}\right)$ and finally cross over to a globally stable phase of black hole for $T>T_{2}$. We collectively identify all these features as being the analog of HP transitions [36] in the "charged" JT model whose holographic interpretation is yet to be unfolded.

The organization for the rest of the paper is as follows: We construct our $D=2$ model in Sec. II, where we give a brief account for the dimensional reduction procedure and obtain the corresponding background solutions following a perturbative approach. Adopting to a Euclidean framework in Sec. III, we explore the thermal stability in black holes using a canonical ensemble. Finally, we conclude in Sec. IV.

\section{II. $D=2$ MODEL}

\section{A. A top down construction}

We consider a $D=5$ gravity model minimally coupled to both Abelian $\left(A_{M}\right)$ as well as $S U(2)$ Yang-Mills (YM) fields $\left[A_{M}^{a}(a=1,2,3)\right][31]$ :

$$
S_{5 D}=\int d^{5} x \sqrt{-g}\left(\mathcal{R}-3 \Lambda-\frac{\kappa}{4 g_{s}^{2}} F_{M N}^{a} F^{a M N}-\frac{\xi}{4} F_{M N} F^{M N}-\frac{\sigma}{2 g_{2}^{2}} \frac{\epsilon^{M N P Q R}}{\sqrt{-g}} F_{M N}^{a} F_{P Q}^{a} A_{R}\right)
$$

where $\Lambda(<0)$ is the cosmological constant. ${ }^{1}$ Here $\kappa$ and $\xi$ are the gauge coupling constants which would be treated as an expansion parameter in the subsequent analysis. The CS piece $\left(\sim F^{a} \wedge F^{a} \wedge A\right)$, on the other hand, is quite ubiquitous to $D=5$ theories and does not seem to have left with any of its imprints on the reduced $D=2$ model.

The $D=2$ theory is obtained using the reduction ansatz [28]

$$
d s^{2}=\Phi^{\alpha} d \tilde{s}^{2}+\Phi^{\beta} d x_{i}^{2}, \quad A_{M}^{a} d x^{M}=A_{\mu}^{a} d x^{\mu}, \quad A_{M} d x^{M}=A_{\mu} d x^{\mu} ; \quad \alpha, \beta \in \mathbb{R},
$$

together with the metric of the reduced space-time,

$$
d \tilde{s}^{2}=\tilde{g}_{\mu \nu} d x^{\mu} d x^{\nu} ; \quad g_{\mu \nu}=\Phi^{\alpha} \tilde{g}_{\mu \nu} ; \quad \mu, \nu=t, z .
$$

The $D=2$ action (modulo a total derivative) could be formally expressed as ${ }^{2}$

$$
\begin{aligned}
S_{2 D} & =\mathcal{V}_{3} \int d^{2} x \sqrt{-\tilde{g}}\left(\tilde{\mathcal{R}} \Phi-V(\Phi)-\Phi \mathcal{L}\left(\tilde{A}_{\mu}, \tilde{A}_{\mu}^{a}\right)\right)+S_{\mathrm{GH}}+S_{\mathrm{ct}}, \\
\mathcal{L}\left(\tilde{A}_{\mu}, \tilde{A}_{\mu}^{a}\right) & =\frac{\kappa}{4 g_{s}^{2}} \tilde{F}_{\mu \nu}^{a} \tilde{F}^{a \mu \nu}+\frac{\xi}{4} \tilde{F}_{\mu \nu} \tilde{F}^{\mu \nu} ; \quad V(\Phi)=3 \Lambda \Phi,
\end{aligned}
$$

where we set $\alpha=0$ and $\beta=2 / 3$ without any loss of generality. Notice that our model (2.4) is a special case of Ref. [17] with $C=0$ and $A=-3 \Lambda$. Moreover, here $S_{\mathrm{GH}}=-\int d t \sqrt{-\gamma} \mathcal{K} \Phi$ is the standard Gibbons-Hawking (GH) term (K being the extrinsic curvature associated with the boundary hypersurface [42]) and $S_{\mathrm{ct}}$ is the so-called counterterm which cures the divergences of the on-shell action near its asymptotic limits.

\footnotetext{
${ }^{1}$ We set the AdS radius $L=1$ and $16 \pi G=1$ in the subsequent analysis.

${ }^{2}$ The methodology of dimensional reduction from $5 D$ to $2 D$ has been briefly discussed in the Appendix A.
} 


\section{B. Equations of motion}

The equations of motion that readily follow from the variation of the parent action (2.4) are listed below:

$$
\begin{aligned}
\left(\nabla_{\mu} \nabla_{\nu}-\tilde{g}_{\mu \nu} \square\right) \Phi+\frac{\xi \Phi}{2}\left(\tilde{F}_{\mu \rho} \tilde{F}_{\nu}^{\rho}-\frac{1}{4} \tilde{F}^{2} \tilde{g}_{\mu \nu}\right) & \\
+\frac{\kappa \Phi}{2 g_{s}^{2}}\left(\tilde{F}_{\mu \rho}^{a} \tilde{F}_{\nu}^{a \rho}-\frac{1}{4} \tilde{F}^{a^{2}} \tilde{g}_{\mu \nu}\right)-\frac{3 \Lambda}{2} \Phi \tilde{g}_{\mu \nu} & =0 \\
\tilde{\mathcal{R}}-3 \Lambda-\frac{\kappa}{4 g_{s}^{2}} \tilde{F}_{\mu \nu}^{a} \tilde{F}^{a \mu \nu}-\frac{\xi}{4} \tilde{F}_{\mu \nu} \tilde{F}^{\mu \nu} & =0 \\
\frac{1}{\sqrt{-\tilde{g}}} \partial_{\mu}\left(\sqrt{-\tilde{g}} \Phi \tilde{F}^{a \mu \nu}\right)+\Phi \epsilon^{a b c} \tilde{A}_{\mu}^{b} \tilde{F}^{c \mu \nu} & =0 \\
\partial_{\mu}\left(\sqrt{-\tilde{g}} \Phi \tilde{F}^{\mu \nu}\right) & =0
\end{aligned}
$$

In order to proceed further, we choose to work with the static metric ansatz

$$
d s^{2}=e^{2 \omega(z)}\left(-d t^{2}+d z^{2}\right)
$$

together with the ansatz for the gauge fields:

$$
\begin{aligned}
& \tilde{A}_{\mu}=\left(\tilde{A}_{t}(z), 0\right), \\
& \tilde{A}_{\mu}^{a}=\tilde{A}_{t}^{3}(z) \tau^{3} d t+\tilde{A}_{z}^{1}(z) \tau^{1} d z,
\end{aligned}
$$

where $\tau^{a}=\sigma^{a} / 2 i$ are the Pauli matrices of $S U(2) \mathrm{YM}$ theory.

Using (2.6), (2.7a), and (2.7b), we finally note down the set of dynamical equations ${ }^{3}$ :

$$
\begin{aligned}
& 2 \Phi^{\prime \prime}+6 \Lambda \Phi e^{2 \omega}+\Phi e^{-2 \omega}\left[\xi\left(\tilde{A}_{t}^{\prime}\right)^{2}+\frac{\kappa}{g_{s}^{2}}\left(\left(\chi^{\prime}\right)^{2}+\chi^{2} \eta^{2}\right)\right]=0, \\
& \Phi^{\prime \prime}-2 \omega^{\prime} \Phi^{\prime}=0, \\
& 4 \omega^{\prime \prime}+6 \Lambda e^{2 \omega}-e^{-2 \omega}\left[\xi\left(\tilde{A}_{t}^{\prime}\right)^{2}+\frac{\kappa}{g_{s}^{2}}\left(\left(\chi^{\prime}\right)^{2}+\chi^{2} \eta^{2}\right)\right]=0, \\
& \Phi \tilde{A}_{t}^{\prime \prime}+\Phi^{\prime} \tilde{A}_{t}^{\prime}-2 \Phi \omega^{\prime} \tilde{A}_{t}^{\prime}=0, \\
& \left(\Phi e^{-2 \omega} \chi \eta\right)^{\prime}+e^{-2 \omega} \Phi \eta \chi^{\prime}=0, \\
& \left(\Phi e^{-2 \omega} \chi^{\prime}\right)^{\prime}-e^{-2 \omega} \Phi \eta^{2} \chi=0
\end{aligned}
$$

\footnotetext{
${ }^{3}$ In order to simplify our notations, we set $\tilde{A}_{t}^{3}=\chi(z)$ and $\tilde{A}_{z}^{1}=\eta(z)$.
}

together with the following constraint:

$$
\Phi e^{-2 \omega} \chi^{2} \eta=0
$$

\section{Solving the dynamics}

We propose the following perturbative method of solving the dynamics ${ }^{4}(2.8 \mathrm{a})-(2.8 \mathrm{f})$ :

$$
\mathcal{A}(z)=\mathcal{A}_{(0)}(z)+\xi \mathcal{A}_{(1)}^{a b}(z)+\kappa \mathcal{A}_{(1)}^{n a}(z)+\cdots,
$$

which is a perturbation in the gauge coupling parameters over the uncharged background solutions [17]. In the present analysis, we retain ourselves only up to leading order in the perturbative expansion (2.10). The general strategy would be to substitute (2.10) into (2.8a)-(2.8f) and obtain equations of motion at different order in the perturbative expansion. ${ }^{5}$ Here, $\mathcal{A}(z)$ stands for either of the dynamical variables $\Phi, \omega, \chi$, and $\eta$.

Before we proceed further, the following observations are noteworthy to mention:

(i) Since we are interested in expanding the action (2.4) up to leading order in the gauge coupling, it is sufficient to estimate leading- or zeroth-order solutions for both Abelian and non-Abelian gauge fields.

(ii) The Abelian sector (2.8d) could be further simplified in terms of other variables,

$$
F_{z t} \equiv A_{t}^{\prime}=\frac{Q e^{2 \omega}}{\Phi},
$$

where $Q$ is the corresponding $U(1)$ charge.

(iii) The dilaton equation of motion (2.8b) could be recast as

$$
\Phi^{\prime}(z)=-\frac{e^{2 \omega}}{2}
$$

whose solution may be expressed as

$$
\Phi(z) \simeq-\int \frac{d z}{2} e^{2 \omega}
$$

\section{Interpolating vacuum}

In order to find vacuum solutions, the first step would be to note down zeroth-order solutions ${ }^{6}$ for both the dilaton and the metric from (B1a) and (B2a):

\footnotetext{
${ }^{4}$ Here $a b$ and $n a$ stand for respective perturbative corrections (to the uncharged JT solutions [17]) due to Abelian and nonAbelian sectors, respectively, of the $D=2$ model (2.4).

${ }_{6}^{5}$ See Appendix B for details.

${ }^{6}$ See Appendix $C$ for the details of the derivation.
} 


$$
\begin{aligned}
e^{2 \omega_{(0)}^{\mathrm{vac}}} & =-\frac{2}{3 \Lambda z^{2}}, \\
\Phi_{(0)}^{\mathrm{vac}} & =-\frac{1}{3 \Lambda z} .
\end{aligned}
$$

Combining (2.14a) and (2.6), it is by now quite evident that the vacuum solution corresponds to $\mathrm{AdS}_{2}$ space-time in standard Poincaré coordinates

$$
d s^{2} \sim z^{-2}\left(-d t^{2}+d z^{2}\right),
$$

where we identify $z \rightarrow 0$ as the asymptotic UV limit of the bulk space-time. On the other hand, $z \rightarrow \infty$ stands for the IR limit [17].

Next, we note down solutions corresponding to (B4a) and (B4b):

$$
\begin{aligned}
& \chi_{(0)}^{\mathrm{vac}} \simeq \log z, \\
& \eta_{(0)}^{\mathrm{vac}} \simeq \frac{1}{z(1+\log z)^{2}},
\end{aligned}
$$

where we have multiplied (B4b) by $\chi_{(0)} / \eta_{(0)}$ and used (B3a).

On the other hand, using (B2b) and (B2c), the solutions corresponding to $\omega_{(1)}^{a b}$ and $\omega_{(1)}^{n a}$ can be found as

$$
\begin{aligned}
& \left(\omega_{(1)}^{a b}\right)^{\mathrm{vac}}=10 z^{2}+\frac{\mathrm{C}}{z}+\frac{Q^{2} \Lambda}{6} z^{2}(1-3 \log z), \\
& \left(\omega_{(1)}^{n a}\right)^{\mathrm{vac}}=-\frac{3 \Lambda}{z} .
\end{aligned}
$$

Finally, the leading-order solutions for dilaton may be obtained from (B1b) and (B1c):

$$
\begin{aligned}
& \left(\Phi_{(1)}^{a b}\right)^{\mathrm{vac}}=\frac{-3\left(\mathrm{C}-20 z^{3}\right)+4 Q^{2} \Lambda z^{3}\left(1-\frac{3}{4} \cdot \log z\right)}{9 \Lambda z^{2}}, \\
& \left(\Phi_{(1)}^{n a}\right)^{\mathrm{vac}} \simeq \frac{1}{z^{2}} .
\end{aligned}
$$

Combining all these pieces together, we find

$$
\begin{aligned}
d s_{\mathrm{vac}}^{2} & =e^{2 \omega^{\mathrm{vac}}}\left(-d t^{2}+d z^{2}\right) \\
& \approx e^{2 \omega_{(0)}^{\mathrm{vac}}}\left(1+2 \xi\left(\omega_{(1)}^{a b}\right)^{\mathrm{vac}}+2 \kappa\left(\omega_{(1)}^{n a}\right)^{\mathrm{vac}}\right)\left(-d t^{2}+d z^{2}\right) .
\end{aligned}
$$

Given the above metric structure (2.20), it is customary to explore various asymptotic limits associated to it. For example, near the IR $(z \rightarrow \infty)$ region, one finds
$e_{\mathrm{IR}}^{2 \omega^{\mathrm{vac}}} \simeq-\frac{2}{3 \Lambda z^{2}}-\frac{2}{9 \Lambda}\left(60 \xi+Q^{2} \xi \Lambda(1-3 \log z)\right)+\mathcal{O}\left(z^{-3}\right)$,

which clearly reveals an emerging $\mathrm{AdS}_{2}$ geometry.

On the other hand, the UV $(z \rightarrow 0)$ limit of the metric reveals

$$
\begin{aligned}
e_{\mathrm{UV}}^{2 \omega \mathrm{vac}} \simeq & \frac{1}{z^{3}}\left(-\frac{4 \xi \mathrm{C}}{3 \Lambda}+4 \kappa\right)-\frac{2}{3 \Lambda z^{2}} \\
& -\frac{2}{9 \Lambda}\left(60 \xi+Q^{2} \xi \Lambda(1-3 \log z)\right)+\mathcal{O}\left(z^{2}\right),
\end{aligned}
$$

an emerging Lifshitz 2 geometry with dynamical exponent $z_{\mathrm{dyn}}=\frac{3}{2}$. A careful analysis further reveals that, for $\xi=\kappa=0$, the resulting geometry becomes $\mathrm{AdS}_{2}$ in both the asymptotic limits. This confirms that gauge fields in the theory are actually responsible for the change in asymptotic (UV) structure as found earlier in Refs. [30,34].

\section{2D black holes}

The zeroth-order or uncharged background solutions $\omega_{(0)}$ and $\Phi_{(0)}$ may be expressed as

$$
\begin{aligned}
e^{2 \omega_{(0)}^{\mathrm{BH}}} & =-\frac{8 \mu}{3 \Lambda \sinh ^{2}(2 \sqrt{\mu} z)}, \\
\Phi_{(0)}^{\mathrm{BH}} & =-\frac{2 \sqrt{\mu}}{3 \Lambda} \operatorname{coth}(2 \sqrt{\mu} z) .
\end{aligned}
$$

Below, we note down first-order solutions corresponding to the metric and the dilaton. In order to simplify our analysis, we consider the following change of coordinate:

$$
z=\frac{1}{2 \sqrt{\mu}} \operatorname{coth}^{-1}\left(\frac{\rho}{\sqrt{\mu}}\right) .
$$

Using (2.24), the solution corresponding to (B2b) can be expressed as

$$
\begin{aligned}
\left(\omega_{(1)}^{a b}\right)^{\mathrm{BH}} \simeq & \frac{\rho}{\sqrt{\mu}}+\left[\frac{\rho}{2 \sqrt{\mu}} \cdot \log \left(\frac{\sqrt{\mu}+\rho}{\sqrt{\mu}-\rho}\right)-1\right] \\
& -\frac{3 Q^{2} \Lambda}{16 \mu^{3 / 2}}\{2 \sqrt{\mu}(1+\log \rho) \\
& +\rho \log \rho \cdot \log \left(\frac{\sqrt{\mu}-\rho}{\sqrt{\mu}+\rho}\right) \\
& \left.+\rho\left[\operatorname{Li}\left(2, \frac{\rho}{\sqrt{\mu}}\right)-\operatorname{Li}\left(2, \frac{-\rho}{\sqrt{\mu}}\right)\right]\right\} .
\end{aligned}
$$

On the other hand, substituting (B2c) in (B1c) and using (2.24), we find 


$$
\left(\omega_{(1)}^{n a}\right)^{\mathrm{BH}} \simeq \frac{\sqrt{\mu} \rho+\left(\mu-\rho^{2}\right) \tanh ^{-1}\left(\frac{\rho}{\sqrt{\mu}}\right)}{2(\mu-1) \mu^{3 / 2}} .
$$

Finally, we note down zeroth-order solutions for gauge fields that readily follow from $(\mathrm{B} 4 \mathrm{a})$ and $(\mathrm{B} 4 \mathrm{~b})$ :

$$
\begin{aligned}
& \chi_{(0)}^{\mathrm{BH}} \simeq 1-\frac{1}{2 \sqrt{\mu}} \log \left(\frac{\rho}{\sqrt{\mu}}\right), \\
& \eta_{(0)}^{\mathrm{BH}} \simeq \frac{\left(\rho^{2}-\mu\right)}{4 \rho \mu^{3 / 2}}\left(1-\frac{1}{\sqrt{2 \mu}} \log \left(\frac{\rho}{\sqrt{\mu}}\right)\right)^{-2} .
\end{aligned}
$$

Collecting all the pieces together, we note down the black hole metric as

$$
\begin{aligned}
d s_{\mathrm{BH}}^{2} \simeq & -\frac{8}{3 \Lambda}\left(\rho^{2}-\mu\right)\left(1+2 \xi\left(\omega_{(1)}^{a b}\right)^{\mathrm{bh}}\right. \\
& \left.+2 \kappa\left(\omega_{(1)}^{n a}\right)^{\mathrm{bh}}\right)\left(-d t^{2}+\frac{d \rho^{2}}{4\left(\rho^{2}-\mu\right)^{2}}\right) .
\end{aligned}
$$

The black hole solution (2.28) exhibits a horizon at $\rho=\sqrt{\mu}$. On the other hand, substituting $\rho \sim \frac{1}{\delta}$ and thereby taking $\delta \rightarrow 0$ limit reveals $\left.d s_{\mathrm{BH}}^{2}\right|_{\rho \rightarrow \infty} \sim \frac{1}{\delta^{3}}$. This suggests that the UV asymptotic of the space-time approaches Lifshitz $_{2}$ geometry with dynamical critical exponent $z_{\text {dyn }}=\frac{3}{2}$.

The Hawking temperature of the black hole (2.28) can be found as

$$
T_{H}=\frac{\sqrt{\mu}}{\pi} .
$$

Finally, we note down the Wald entropy [43] of the black hole $^{7}$ :

$$
\begin{aligned}
S_{W}= & \left.4 \pi \Phi\right|_{\rho=\sqrt{\mu}} \\
= & -\frac{1}{96 \Lambda \sqrt{\mu}(|\mu-1|)}\left[( | \mu - 1 | ) \left\{-33 Q^{2} \Lambda \xi\right.\right. \\
& \left.\left.+64 \mu(1+0.89 \xi)-15 Q^{2} \xi(\log \mu)\right\}-240 \kappa\right],
\end{aligned}
$$

which clearly shows a discontinuity at $\mu=\mu_{0}=1$. As we probe into details on thermal stability, we identify the above discontinuity as a signature of a first-order transition from an unstable black hole phase to pure radiation.

In order to compare the $2 D$ black hole entropy (2.30) with the black hole entropy in $5 D$, we uplift the $2 D$ solution into $5 D^{\prime \prime}$

$$
d s_{5 D}^{2}=d s_{2 D}^{2}+\Phi^{2 / 3} d x_{i}^{2}, \quad i=1,2,3 .
$$

\footnotetext{
${ }^{7}$ Here by entropy we mean entropy per unit volume of the transverse space, namely, $S_{W} / \mathcal{V}_{3}$.
}

In a manner similar to the $2 D$ theory, the Wald entropy corresponding to the $5 D$ black hole can be written as $[44,45]$

$$
\begin{aligned}
\hat{S}_{W} & =-2 \pi \int_{\Sigma}\left(\frac{\delta \mathcal{L}_{5 D}}{\delta \mathcal{R}_{a b c d}}\right) \hat{\epsilon}_{a b} \hat{\epsilon}_{c d} \bar{\epsilon} \\
& =-2 \pi \int_{\rho=\sqrt{\mu}, t=\mathrm{const}}\left(\frac{\delta \mathcal{L}_{5 D}}{\delta \mathcal{R}_{\rho t \rho t}}\right) \hat{\epsilon}_{\rho t} \hat{\epsilon}_{\rho t} \Phi \prod_{i=1}^{3} d x_{i} \\
& =\left.\mathcal{V}_{3} 4 \pi \Phi\right|_{\rho=\sqrt{\mu}},
\end{aligned}
$$

where $\Sigma$ is the codimension-2 spacelike bifurcation surface with binormal $\hat{\epsilon}_{a b}$ which is symmetric in $a \leftrightarrow b$ and normalized as $\hat{\epsilon}^{a b} \hat{\epsilon}_{a b}=-2, \bar{\epsilon}$ is the induced volume on $\Sigma$, and $\mathcal{R}_{a b c d}$ is the Riemann curvature tensor in the theory. Also, the bifurcation surface is at the horizon $\rho=\sqrt{\mu}$, $t=$ const. Notice that, in deriving the final form of the Wald entropy in (2.32), we have used the fact that the Lagrangian density $\mathcal{L}_{5 D}$ corresponding to the $5 D$ theory (2.1) contains a term linear in $\mathcal{R}$ only. In addition, we have used the form of the metric given in the ansatz (2.2) explicitly. Hence, from (2.30) and (2.32), we observe that the Wald entropies [per unit volume of the transverse space $\left.\left(\mathcal{V}_{3}\right)\right]$ for the two theories indeed match.

\section{THERMAL STABILITY AND HP TRANSITION}

We now move on to the Euclidean formalism [46] and explore thermal stability in black holes. To start with, the full Euclidean on-shell action is schematically expressed as

$$
-S_{2 D}^{\mathrm{os}}=S_{\mathrm{grav}}^{\mathrm{os}}+S_{\mathrm{GH}}^{\mathrm{os}}+S_{\mathrm{ct}}^{\mathrm{os}},
$$

where each of the above entities on the rhs of (3.1) are estimated using background solutions found in the previous section. This is accompanied by an analytic continuation of the Lorentzian time, $t \rightarrow i \tau$ with a periodicity $\beta$. In the present analysis, there exist two distinct periodicities corresponding to the thermal radiation bath $\left(\beta_{\mathrm{TH}}\right)$ and the black hole phase $\left(\beta_{\mathrm{BH}}\right)$, respectively. The black hole periodicity $\beta_{\mathrm{BH}}$ is uniquely fixed by (2.29). On the other hand, the former $\left(\beta_{\mathrm{TH}}\right)$ could be fixed by demanding that the ratio

$$
\frac{\beta_{\mathrm{TH}}}{\beta_{\mathrm{BH}}} \approx 2+\mathcal{O}\left(\frac{1}{\rho_{c}}\right)
$$

becomes independent of the radial cutoff $\rho=\rho_{c}(\rightarrow \infty)$ at large distances [46].

Using (3.2), the effective free energy of the configuration could be obtained using the background subtraction method [46]: 


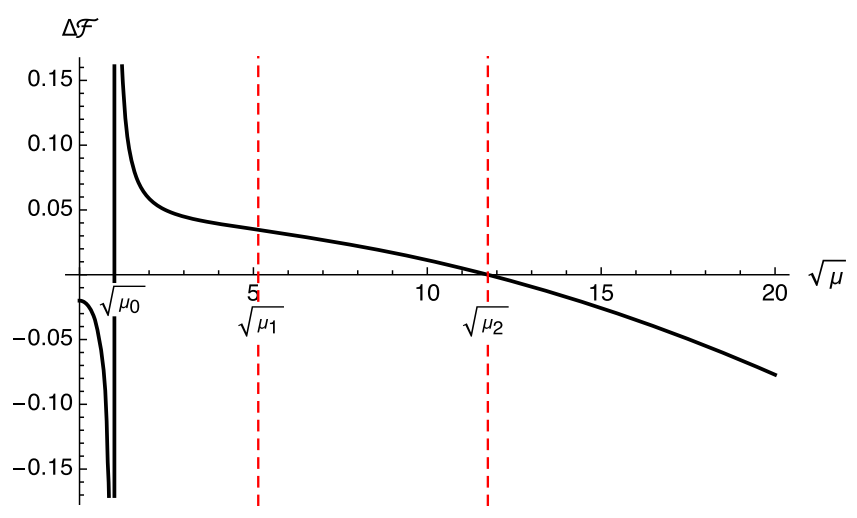

(a) Free energy vs. temperature plot.

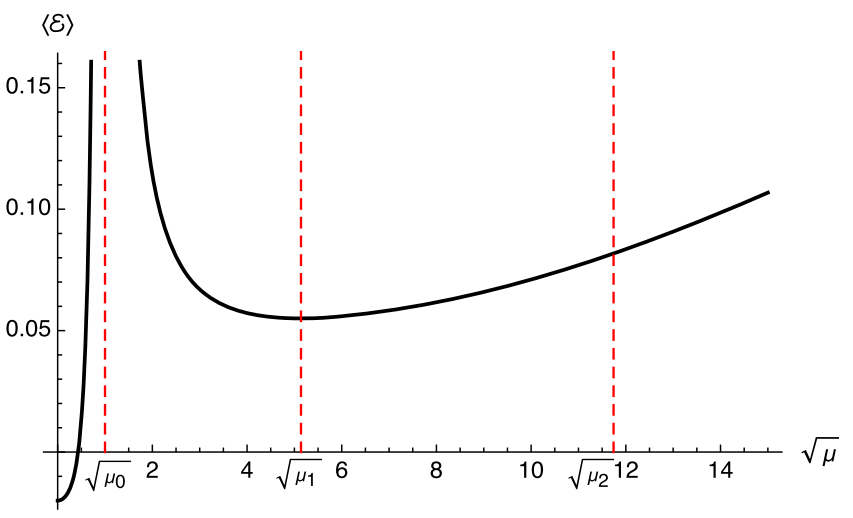

(b) Energy ( Mass) vs. temperature plot.

FIG. 1. Behavior of effective free energy $(\Delta \mathcal{F})$ and energy $\left(\mathcal{E} \sim \mathcal{M}_{\mathrm{BH}}\right)$ of the configuration with temperature $T(\sim \sqrt{\mu})$.

$$
\begin{aligned}
\Delta \mathcal{F}= & \mathcal{F}_{\mathrm{BH}}-\mathcal{F}_{\mathrm{TH}} \\
= & \frac{1}{18 \Lambda(\mu-1)}\left[( \mu - 1 ) \left\{-7 Q^{2} \Lambda \xi\right.\right. \\
& +16 \xi(-30+0.22 \mu)\}+120 \Lambda \kappa] .
\end{aligned}
$$

Notice that this free energy is quite unique to our original choices of parameters $\alpha$ and $\beta$ in (2.2) and, therefore, to the JT gravity model proposed in (2.4). In other words, the background subtraction method used in this paper is also quite pertinent to the choices of parameters $\alpha$ and $\beta$.

Finally, using a canonical ensemble $\left(\mathcal{Z} \sim e^{-\Delta S_{2 D}^{\text {os }}}\right)$, the energy of the black hole configuration may be estimated as

$$
\begin{aligned}
\mathcal{M}_{\mathrm{BH}} \equiv & \langle\mathcal{E}\rangle=\frac{1}{450 \Lambda(\mu-1)^{2}}(3000 \kappa \Lambda(3 \mu-1) \\
& \left.-\xi(\mu-1)^{2}\left(12000+175 Q^{2} \Lambda+88 \mu\right)\right) .
\end{aligned}
$$

Figure 1 displays the behavior of both the free energy $(\Delta \mathcal{F})$ and the energy ( $\sim$ mass) of the (black hole) configuration with temperature $(T \sim \sqrt{\mu})$. In all the subsequent plots, we set $Q=0.01, \xi=0.009$, and $\kappa=0.009$. Below, we enumerate the key observations regarding Figs. 1 and 2.

(i) To start with, we notice that the thermal radiation collapses to form an unstable black hole in the region $T_{0}<T<T_{1}$ which can either completely decay into pure radiation without a black hole (for $T<T_{0}$ ) or transit to a larger mass black hole for $T>T_{0}$. Clearly, in this phase, the smaller mass black hole has larger free energy than the thermal radiation bath $\left(\mathcal{F}_{\mathrm{BH}}>\mathcal{F}_{\mathrm{TH}}\right)$ and is also characterized by a negative heat capacity $C_{\mathrm{BH}} \sim \frac{\partial\langle\mathcal{E}\rangle}{\partial \sqrt{\mu}}<0$ [Fig. 1(b)]. Therefore, we identify $T_{0}<T<T_{1}$ as a radiation-dominated phase with $\Delta \mathcal{F}>0$ and $T<T_{0}$ as a pure radiation phase with $\Delta \mathcal{F} \sim \mathcal{F}_{\mathrm{TH}}<0$
[Fig. 1(a)]. The corresponding entropy ${ }^{8}$ plot (Fig. 2) reveals the onset of a first-order phase transition at $T_{0} \sim \sqrt{\mu_{0}}=1$. The $\sqrt{\mu}<1$ branch [Fig. 2(a)] corresponds to entropy of the configuration that ultimately boils down into pure radiation. This part of the phase diagram represents a rapidly evaporating black hole [Fig. 1(b)] placed in a heat bath which eventually results in a thermal ensemble with unique entropy $\left(\mathcal{S}_{\mathrm{TH}}\right)$. The $\sqrt{\mu}>1$ branch [Fig. 2(b)], on the other hand, depicts a phase with decreasing difference entropy $(\Delta \mathcal{S})$ for $T \gtrsim T_{0}$ which corresponds to the fact that the entropy of the unstable black hole approaches the entropy of the thermal bath as it gradually shrinks in size [Fig. 1(b)] with the increase in temperature. This, in turn, is related to the negative heat capacity of the black hole as mentioned earlier.

(ii) As the temperature of the configuration approaches $T \sim T_{1} \sim \sqrt{\mu_{1}}$, the corresponding mass of the black hole reaches a minima [Fig. 1(b)] and thereafter starts increasing slowly for $T \gtrsim T_{1}$. We identify this as a locally ${ }^{9}$ stable phase of a black hole $\left(\mathcal{S}_{\mathrm{BH}} \sim \mathcal{S}_{\mathrm{TH}}\right)$ with positive heat capacity $C_{\mathrm{BH}} \sim$ $\frac{\partial\langle\mathcal{E}\rangle}{\partial \sqrt{\mu}}>0$ [Fig. 1(b)]. However, the effective free energy $(\Delta \mathcal{F})$ of the configuration reveals that this

\footnotetext{
${ }^{8}$ The entropy $(\mathcal{S})$ that we measure should be understood in two ways. For the $\sqrt{\mu}<1$ branch, one is left with pure radiation; therefore, we identify the corresponding entropy simply as the entropy of the thermal bath $\left(\mathcal{S}_{\mathrm{TH}}\right)$. On the other hand, for the $\sqrt{\mu}>1$ branch, we introduce the notion of effective (or difference) entropy, $\Delta \mathcal{S} \sim-\frac{\Delta \mathcal{F}}{\Delta \sqrt{\mu}} \sim\left|\mathcal{S}_{\mathrm{BH}}-\mathcal{S}_{\mathrm{TH}}\right|$, which is basically the difference in entropy between two distinct configurations, namely, the black hole and the heat bath surrounding it [see (3.3)]. However, in both cases, the change in total entropy must be positive following the second law of thermodynamics.

${ }^{9}$ By local we mean that this phase is existing only in a small temperature window $T_{1}<T<T_{2}$.
} 


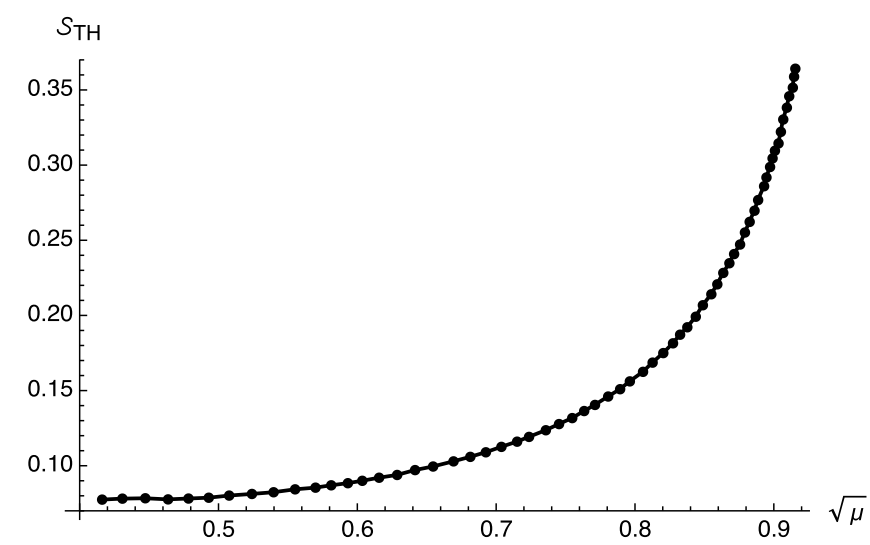

(a) Thermal entropy $\left(\mathcal{S}_{\mathrm{TH}}\right)$ plot for $\sqrt{\mu}<1$ branch.

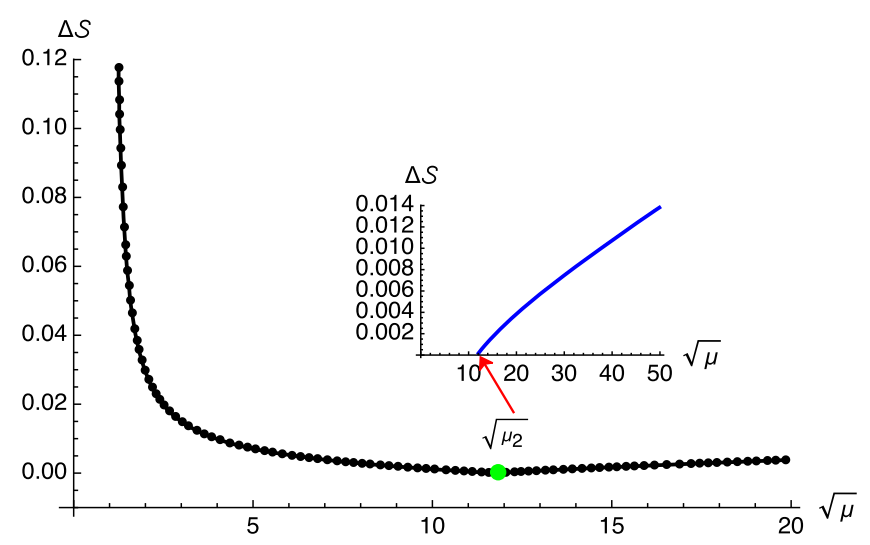

(b) Effective entropy $(\Delta \mathcal{S})$ plot for the $\sqrt{\mu}>1$ branch.

FIG. 2. Behavior of the entropy $(\mathcal{S})$ as a function of temperature $T(\sim \sqrt{\mu})$.

phase is still dominated by the thermal radiation background with $\mathcal{F}_{\mathrm{TH}}<\mathcal{F}_{\mathrm{BH}}$ [Fig. 1(a)]. The total entropy of the configuration is therefore $\sim \mathcal{S}_{\mathrm{TH}}$ and, hence, the difference $\Delta \mathcal{S} \sim 0$ over the range $T_{1}<T<T_{2}$ [Fig. 2(b)].

(iii) Finally, we reach the point of crossover $T=T_{2}$ beyond which the black hole mass $\left(\mathcal{M}_{\mathrm{BH}}\right)$ starts becoming ever increasing with the increase in temperature and thereby yields a positive heat capacity $\left(C_{\mathrm{BH}}>0\right)$ [Fig. 1(b)]. A careful analysis further reveals that this is the point of inflection where the difference entropy vanishes $(\Delta \mathcal{S}=0)$ and thereafter starts increasing [Fig. 2(b)] as one approaches the region $T>T_{2}$. This corresponds to a globally ${ }^{10}$ stable phase of black hole with $\mathcal{F}_{\mathrm{BH}}<$ $\mathcal{F}_{\text {TH }}$ [Fig. 1(a)]. Needless to say, for $T>T_{2}$, the entropy content of the configuration is mostly dominated due to the presence of the larger mass black hole which largely contributes to both in the increase in total entropy as well as the difference entropy $(\Delta \mathcal{S} \gtrsim 0)$ of the system.

\section{SUMMARY AND FINAL REMARKS}

We conclude with a brief summary of the main outcome in this analysis. We propose a possible formulation of $D=2$ Jackiw-Teitelboim (JT) gravity using dimensional reduction of the parent $D=5$ gravity model [31] in the presence of Abelian as well $S U(2)$ Yang-Mills fields. To our surprise, we notice an early (thermal) instability associated to black hole microstates using a canonical ensemble for the $D=2$ model. We identify that these early black hole states might either decay into pure radiation or switch over to bigger black hole microstates with higher

\footnotetext{
${ }^{10} \mathrm{By}$ global we mean that this phase persists for all temperatures $T>T_{2}$.
}

entropy and lesser free energy. We summarize all these features collectively as being the analog of the HawkingPage transition [36] in 2D gravity models. This observation also raises a natural question regarding the holographic interpretation of our analysis in terms of (dual) quantum mechanical DOF living in one dimension. We leave this as an exciting direction to be explored in the near future.

\section{ACKNOWLEDGMENTS}

The work of A. L. is funded by the National Agency for Research and Development (ANID)/FONDECYT/ POSTDOCTORADO BECAS CHILE Project No. 3190021. H. R. and D. R. are indebted to the authorities of Indian Institute of Technology Roorkee for their unconditional support toward research in basic sciences.

\section{APPENDIX A: DIMENSIONAL REDUCTION FROM 5D TO $2 D$}

In order to analyze the dimensional reduction of the $5 D$ theory (2.1) to $2 D$, in the following we rewrite the ansatz given in (2.2):

$$
\begin{aligned}
d s^{2} & =\Phi^{\alpha} d \tilde{s}^{2}+\Phi^{\beta} d x_{i}^{2}, \quad A_{M}^{a} d x^{M}=A_{\mu}^{a} d x^{\mu}, \\
A_{M} d x^{M} & =A_{\mu} d x^{\mu} ; \quad \alpha, \beta \in \mathbb{R} .
\end{aligned}
$$

With this choice of the metric, we note that

$$
\sqrt{-g}=\sqrt{-\tilde{g}} \Phi^{\alpha+3 \beta / 2}
$$

where $\tilde{g}$ is the determinant of the two-dimensional metric.

It is then easy to check that the first two terms in the action (2.1) can be written as

$$
\sqrt{-g}(\mathcal{R}-3 \Lambda)=\sqrt{-\tilde{g}} \Phi^{3 \beta / 2}\left(\tilde{\mathcal{R}}-3 \Lambda \Phi^{\alpha}+\Phi^{\alpha} g^{i j} \mathcal{R}_{i j}\right) .
$$


In the next step, we rewrite the third term within the curly braces in (A3) as a total derivative term given by

$$
\Phi^{\alpha+3 \beta / 2} g^{i j} \mathcal{R}_{i j} \sim \nabla_{\mu}\left[g^{\mu \nu} g^{\lambda \sigma} g_{\lambda \sigma}\left(\partial_{\nu} \Phi^{\beta}\right) \Phi^{\alpha+\beta / 2}\right],
$$

which therefore does not contribute to the bulk equations of motion (2.5a)-(2.5d). Notice that the JT gravity model (2.4) can be obtained once we set the parameters $\alpha=0$ and $\beta=2 / 3$. In other words, these choices of parameters are quite pertinent to the JT gravity model discussed in this paper. The remaining terms in (2.4) involving gauge fields can be obtained in a similar manner.

\section{APPENDIX B: EQUATIONS OF MOTION AT DIFFERENT ORDERS IN THE PERTURBATION SERIES}

In the following, we note down the equations of motion up to leading order in perturbative expansion. For example, substituting (2.10) into (2.8), we obtain

$$
\begin{aligned}
\mathcal{O}(0): & 2 \Phi_{(0)}^{\prime \prime}+6 \Lambda \Phi_{(0)} e^{2 \omega_{(0)}}=0, \\
\mathcal{O}(\xi): & 2\left(\Phi_{(1)}^{a b}\right)+6 \Lambda e^{2 \omega_{(0)}}\left(2 \omega_{(1)}^{a b} \Phi_{(0)}+\Phi_{(1)}^{a b}\right) \\
& +\frac{Q^{2}}{\Phi_{(0)}} e^{2 \omega_{(0)}}=0, \\
\mathcal{O}(\kappa): & 2\left(\Phi_{(1)}^{n a}\right)^{\prime \prime}+6 \Lambda e^{2 \omega_{(0)}}\left(2 \omega_{(1)}^{n a} \Phi_{(0)}+\Phi_{(1)}^{n a}\right) \\
& +\frac{1}{g_{s}^{2}} \Phi_{(0)} e^{-2 \omega_{(0)}}\left(\left(\chi_{(0)}^{\prime}\right)^{2}+\chi_{(0)}^{2} \eta_{(0)}^{2}\right)=0 .
\end{aligned}
$$

Similarly, (2.8c) could be arranged up to leading order in the perturbative expansion as

$$
\begin{gathered}
\mathcal{O}(0): 4 \omega_{(0)}^{\prime \prime}+6 \Lambda e^{2 \omega_{(0)}}=0, \\
\mathcal{O}(\xi): 4\left(\omega_{(1)}^{a b}\right)^{\prime \prime}+12 \Lambda \omega_{(1)}^{a b} e^{2 \omega_{(0)}}-\frac{Q^{2}}{\Phi_{(0)}^{2}} e^{2 \omega_{(0)}}=0, \\
\mathcal{O}(\kappa): 4\left(\omega_{(1)}^{n a}\right)^{\prime \prime}+12 \Lambda \omega_{(1)}^{n a} e^{2 \omega_{(0)}}-\frac{e^{-2 \omega_{(0)}}}{g_{s}^{2}} \\
+\left(\left(\chi_{(0)}^{\prime}\right)^{2}+\chi_{(0)}^{2} \eta_{(0)}^{2}\right)=0 .
\end{gathered}
$$

Substituting (2.10) into the constraint equation (2.9), we obtain

$$
\begin{aligned}
\mathcal{O}(0): & \Phi_{(0)} e^{-2 \omega_{(0)}} \chi_{(0)}^{2} \eta_{(0)}=0 \\
\mathcal{O}(\xi): & \Phi_{(0)}\left(\chi_{(0)} \eta_{(1)}^{a b}+2 \eta_{(0)} \chi_{(1)}^{a b}\right) \\
& +\chi_{(0)} \eta_{(0)}\left(\Phi_{(1)}^{a b}-2 \Phi_{(0)} \omega_{(1)}^{a b}\right)=0, \quad e^{-2 \omega_{(0)}} \chi_{(0)}^{2}=0
\end{aligned}
$$

$$
\begin{aligned}
\mathcal{O}(\kappa): & \Phi_{(0)}\left(\chi_{(0)} \eta_{(1)}^{n a}+2 \eta_{(0)} \chi_{(1)}^{n a}\right) \\
& +\chi_{(0)} \eta_{(0)}\left(\Phi_{(1)}^{n a}-2 \Phi_{(0)} \omega_{(1)}^{n a}\right)=0, \quad e^{-2 \omega_{(0)}} \chi_{(0)}^{2}=0 .
\end{aligned}
$$

Finally, from (2.8e) and (2.8f), the zeroth-order equations could be recast as

$$
\begin{gathered}
\partial_{z}\left(\Phi_{(0)} e^{-2 \omega_{(0)}} \chi_{(0)} \eta_{(0)}\right)+\Phi_{(0)} e^{-2 \omega_{(0)}} \eta_{(0)}\left(\partial_{z} \chi_{(0)}\right)=0 \\
\partial_{z}\left(\Phi_{(0)} e^{-2 \omega_{(0)}}\left(\partial_{z} \chi_{(0)}\right)\right)-\Phi_{(0)} e^{-2 \omega_{(0)}} \chi_{(0)} \eta_{(0)}^{2}=0
\end{gathered}
$$

\section{APPENDIX C: DERIVATIONS OF ZEROTH-ORDER SOLUTIONS}

In the conformal gauge [17]

$$
d s^{2}=-e^{2 \omega\left(x^{+}, x^{-}\right)} d x^{+} d x^{-}, \quad x^{ \pm}=(t \pm z),
$$

the zeroth-order equations of motion for the metric and the dilaton can be rewritten as

$$
\begin{gathered}
4 \partial_{+} \partial_{-} \Phi_{(0)}-3 \Lambda \Phi_{(0)} e^{2 \omega_{(0)}}=0, \\
8 \partial_{+} \partial_{-} \omega_{(0)}-3 \Lambda e^{2 \omega_{(0)}}=0, \\
\partial_{+}\left(e^{2 \omega_{(0)}} \partial_{+} \Phi_{(0)}\right)=0, \\
\partial_{-}\left(e^{2 \omega_{(0)}} \partial_{-} \Phi_{(0)}\right)=0 .
\end{gathered}
$$

Clearly, the solution of (C3) is given by

$$
e^{2 \omega_{(0)}}=\left(-\frac{8}{3 \Lambda}\right) \frac{1}{\left(x^{+}-x^{-}\right)^{2}} .
$$

Now integrating (C4) and (C5), we may write

$$
\begin{aligned}
& \partial_{+} \Phi_{(0)}=\left(-\frac{8}{3 \Lambda}\right) \frac{f\left(x^{-}\right)}{\left(x^{+}-x^{-}\right)^{2}}, \\
& \partial_{-} \Phi_{(0)}=\left(-\frac{8}{3 \Lambda}\right) \frac{g\left(x^{+}\right)}{\left(x^{+}-x^{-}\right)^{2}} .
\end{aligned}
$$

Differentiating (C7) with respect to $x^{-}$and (C8) with respect to $x^{+}$and substituting them in $(\mathrm{C} 2)$, we obtain

$$
\Phi_{(0)}=\left\{\begin{array}{l}
\frac{2}{3 \Lambda} \frac{\partial_{-} f\left(x^{-}\right) \cdot\left(x^{+}-x^{-}\right)+2 f\left(x^{-}\right)}{\left(x^{+}-x^{-}\right)} \\
\frac{2}{3 \Lambda} \frac{\partial_{+} g\left(x^{+}\right) \cdot\left(x^{+}-x^{-}\right)-2 g\left(x^{+}\right)}{\left(x^{+}-x^{-}\right)} .
\end{array}\right.
$$

After a few easy algebraic steps, the general solution for the dilaton may be written as [17] 


$$
\Phi_{(0)}=\left(-\frac{2}{3 \Lambda}\right) \frac{a+b\left(x^{+}+x^{-}\right)+c x^{+} x^{-}}{\left(x^{+}-x^{-}\right)}
$$

where $a, b$, and $c$ are real constants. For the vacuum, we may choose $a=1, b=0$, and $c=0[17,25]$.

In the next step, we find the corresponding solutions for the black hole by exploiting the $S L(2, \mathbb{R})$ invariance of the metric $(\mathrm{C} 1)$. The general solutions can then be written as

$$
\begin{aligned}
e^{2 \omega_{(0)}} & =\left(-\frac{8}{3 \Lambda}\right) \frac{\omega^{+}\left(x^{+}\right) \omega^{-}\left(x^{-}\right)}{\left[\omega^{+}\left(x^{+}\right)-\omega^{-}\left(x^{-}\right)\right]^{2}} \\
\Phi_{(0)} & =\left(-\frac{2}{3 \Lambda}\right) \frac{1-\mu \omega^{+}\left(x^{+}\right) \omega^{-}\left(x^{-}\right)}{\left[\omega^{+}\left(x^{+}\right)-\omega^{-}\left(x^{-}\right)\right]}
\end{aligned}
$$

where $\mu(>0)$ can be considered as the mass of the black hole and $\omega^{ \pm}\left(x^{ \pm}\right)$are monotonic functions [17].

Finally, using the conformal transformations

$$
\omega^{ \pm}\left(x^{ \pm}\right)=\frac{1}{\sqrt{\mu}} \tanh \sqrt{\mu} x^{ \pm},
$$

(C11) and (C12) can be rewritten, respectively, as

$$
\begin{gathered}
e^{2 \omega_{(0)}}=\left(-\frac{8}{3 \Lambda}\right) \frac{\mu}{\sinh ^{2}\left(\sqrt{\mu}\left(x^{+}-x^{-}\right)\right)}, \\
\Phi_{(0)}=\left(-\frac{2}{3 \Lambda}\right) \sqrt{\mu} \operatorname{coth}\left(\sqrt{\mu}\left(x^{+}-x^{-}\right)\right) .
\end{gathered}
$$

[1] J. M. Maldacena, The Large N limit of superconformal field theories and supergravity, Int. J. Theor. Phys. 38, 1113 (1999); Adv. Theor. Math. Phys. 2, 231 (1998).

[2] E. Witten, Anti-de Sitter space and holography, Adv. Theor. Math. Phys. 2, 253 (1998).

[3] L. Susskind, The World as a hologram, J. Math. Phys. (N.Y.) 36, 6377 (1995).

[4] A. Kitaev, Hidden correlations in the Hawking radiation and thermal noise, Proceedings of the Fundamental Physics Prize Symposium, November 10, Santa Barbara, U.S.A. (2014).

[5] A. Kitaev, A simple model of quantum holography, Proceedings of the KITP strings seminar and Entanglementprogram, February 12, April 7, and May 27, Santa Barbara, U.S.A. (2015).

[6] S. Sachdev and J. Ye, Gapless Spin Fluid Ground State in a Random, Quantum Heisenberg Magnet, Phys. Rev. Lett. 70, 3339 (1993).

[7] J. Polchinski and V. Rosenhaus, The spectrum in the Sachdev-Ye-Kitaev model, J. High Energy Phys. 04 (2016) 001.

[8] J. Maldacena and D. Stanford, Remarks on the SachdevYe-Kitaev model, Phys. Rev. D 94, 106002 (2016).

[9] C. Teitelboim, Gravitation and Hamiltonian structure in two space-time dimensions, Phys. Lett. B 126, 41 (1983).

[10] R. Jackiw, Lower dimensional gravity, Nucl. Phys. B252, 343 (1985).

[11] A. Jevicki, K. Suzuki, and J. Yoon, Bi-local holography in the SYK model, J. High Energy Phys. 07 (2016) 007.

[12] D. J. Gross and V. Rosenhaus, A generalization of SachdevYe-Kitaev, J. High Energy Phys. 02 (2017) 093.

[13] D. J. Gross and V. Rosenhaus, The bulk dual of SYK: Cubic couplings, J. High Energy Phys. 05 (2017) 092.

[14] A. Kitaev and S. J. Suh, The soft mode in the SachdevYe-Kitaev model and its gravity dual, J. High Energy Phys. 05 (2018) 183.

[15] M. Taylor, Generalized conformal structure, dilaton gravity and SYK, J. High Energy Phys. 01 (2018) 010.
[16] U. Moitra, S. K. Sake, S. P. Trivedi, and V. Vishal, Jackiwteitelboim model coupled to conformal matter in the semiclassical limit, J. High Energy Phys. 04 (2020) 199.

[17] A. Almheiri and J. Polchinski, Models of $\mathrm{AdS}_{2}$ backreaction and holography, J. High Energy Phys. 11 (2015) 014.

[18] J. Maldacena, D. Stanford, and Z. Yang, Conformal symmetry and its breaking in two dimensional Nearly Antide-Sitter space, Prog. Theor. Exp. Phys. 2016, 12C104 (2016).

[19] M. Cvetič and I. Papadimitriou, $\operatorname{AdS}_{2}$ holographic dictionary, J. High Energy Phys. 12 (2016) 008; Erratum, J. High Energy Phys. 01 (2017) 120.

[20] J. Engelsöy, T. G. Mertens, and H. Verlinde, An investigation of $\mathrm{AdS}_{2}$ backreaction and holography, J. High Energy Phys. 07 (2016) 139.

[21] K. Jensen, Chaos in $\mathrm{AdS}_{2}$ Holography, Phys. Rev. Lett. 117, 111601 (2016).

[22] S. R. Das, A. Jevicki, and K. Suzuki, Three dimensional view of the SYK/AdS duality, J. High Energy Phys. 09 (2017) 017.

[23] S. R. Das, A. Ghosh, A. Jevicki, and K. Suzuki, Three dimensional view of arbitrary $q$ SYK models, J. High Energy Phys. 02 (2018) 162.

[24] S. R. Das, A. Ghosh, A. Jevicki, and K. Suzuki, Space-time in the SYK model, J. High Energy Phys. 07 (2018) 184.

[25] H. Kyono, S. Okumura, and K. Yoshida, Deformations of the Almheiri-Polchinski model, J. High Energy Phys. 03 (2017) 173.

[26] A. Lala and D. Roychowdhury, SYK/AdS duality with Yang-Baxter deformations, J. High Energy Phys. 12 (2018) 073.

[27] D. Roychowdhury, Holographic derivation of $q$ SYK spectrum with Yang-Baxter shift, Phys. Lett. B 797, 134818 (2019).

[28] R. A. Davison, W. Fu, A. Georges, Y. Gu, K. Jensen, and S. Sachdev, Thermoelectric transport in disordered metals without quasiparticles: The Sachdev-Ye-Kitaev models and holography, Phys. Rev. B 95, 155131 (2017). 
[29] A. Gaikwad, L. K. Joshi, G. Mandal, and S. R. Wadia, Holographic dual to charged SYK from 3D Gravity and Chern-Simons, J. High Energy Phys. 02 (2020) 033.

[30] A. Lala and D. Roychowdhury, Models of phase stability in Jackiw-Teitelboim gravity, Phys. Rev. D 100, 124061 (2019).

[31] Z. Y. Fan and H. Lü, Electrically-charged Lifshitz spacetimes, and hyperscaling violations, J. High Energy Phys. 04 (2015) 139.

[32] Z. Y. Fan and H. Lu, Charged black holes in colored Lifshitz spacetimes, Phys. Lett. B 743, 290 (2015).

[33] S. Kachru, X. Liu, and M. Mulligan, Gravity duals of Lifshitz-like fixed points, Phys. Rev. D 78, 106005 (2008).

[34] M. Taylor, Non-relativistic holography, arXiv:0812.0530.

[35] L. Romans, Gauged $N=4$ supergravities in fivedimensions and their magnetovac backgrounds, Nucl. Phys. B267, 433 (1986).

[36] S. W. Hawking and D. N. Page, Thermodynamics of black holes in anti-de Sitter space, Commun. Math. Phys. 87, 577 (1983).

[37] A. Chamblin, R. Emparan, C. V. Johnson, and R. C. Myers, Charged AdS black holes and catastrophic holography, Phys. Rev. D 60, 064018 (1999).
[38] A. Chamblin, R. Emparan, C. V. Johnson, and R. C. Myers, Holography, thermodynamics and fluctuations of charged AdS black holes, Phys. Rev. D 60, 104026 (1999).

[39] S. Carlip and S. Vaidya, Phase transitions and critical behavior for charged black holes, Classical Quantum Gravity 20, 3827 (2003).

[40] R. G. Cai, S. P. Kim, and B. Wang, Ricci flat black holes and Hawking-Page phase transition in Gauss-Bonnet gravity and dilaton gravity, Phys. Rev. D 76, 024011 (2007).

[41] Y.S. Myung, Phase transition between non-extremal and extremal Reissner-Nordstrom black holes, Mod. Phys. Lett. A 23, 667 (2008).

[42] G. Gibbons and S. Hawking, Action integrals and partition functions in quantum gravity, Phys. Rev. D 15, 2752 (1977).

[43] R. M. Wald, Black hole entropy is the Noether charge, Phys. Rev. D 48, R3427 (1993).

[44] T. Jacobson, G. Kang, and R. C. Myers, On black hole entropy, Phys. Rev. D 49, 6587 (1994).

[45] R. Brustein, D. Gorbonos, and M. Hadad, Wald's entropy is equal to a quarter of the horizon area in units of the effective gravitational coupling, Phys. Rev. D 79, 044025 (2009).

[46] E. Witten, Anti-de Sitter space, thermal phase transition, and confinement in gauge theories, Adv. Theor. Math. Phys. 2, 505 (1998). 